Research Article

\title{
Observation of Spin Reorientation Transitions in Lead and Titanium-Modified $\mathrm{BiFeO}_{3}$ Multiferroics
}

\author{
Ashwini Kumar $\mathbb{D}^{1}{ }^{1}$ Poorva Sharma ${ }^{\mathbb{D}},{ }^{1}$ Qi Li, ${ }^{2}$ Fujun Qiu, ${ }^{1}$ Jianhui Yan, ${ }^{1}$ Jingyou Tang, \\ and Guolong $\operatorname{Tan}^{1,3}$ \\ ${ }^{1}$ Key Laboratory of Multifunctional Materials, School of Electrical and Electronics Engineering, \\ Luzhou Vocational and Technical College, Luzhou, Sichuan-646000, China \\ ${ }^{2}$ School of Physics, Southeast University, Nanjing-211189, China \\ ${ }^{3}$ Institute of New Materials, Wuhan University of Technology, Wuhan-430070, China \\ Correspondence should be addressed to Ashwini Kumar; ashu.dhanda2@hotmail.com and Poorva Sharma; poorva@nuaa.edu.cn
}

Received 6 May 2021; Accepted 23 September 2021; Published 14 October 2021

Academic Editor: Alicia E. Ares

Copyright (c) 2021 Ashwini Kumar et al. This is an open access article distributed under the Creative Commons Attribution License, which permits unrestricted use, distribution, and reproduction in any medium, provided the original work is properly cited.

\begin{abstract}
We report the synthesis and basic characterization details of bulk $\mathrm{Bi}_{1-x} \mathrm{~Pb}_{x} \mathrm{Fe}_{1-x} \mathrm{Ti}_{x} \mathrm{O}_{3}(x=0.05$ and 0.1$)$ polycrystalline samples, which have been synthesized using the conventional solid-state route. We studied the effects of partially doping of $\mathrm{Pb}$ and $\mathrm{Ti}$ ion on structural, vibrational, and magnetic properties of multiferroic $\mathrm{BiFeO}_{3}$. X-ray diffraction (XRD) was used for crystallographic studies, followed by Rietveld refinement, and phase formation of the compounds was confirmed, which indicates that the sample has rhombohedral $(R 3 c, 100 \%)$ symmetry for $x=0.05$ and $R 3 c(98 \%)+P 4 m m(2 \%)$ symmetry for $x=0.1$. X-ray absorption spectroscopy has been probed at $\mathrm{Fe} L_{2,3}$ and $\mathrm{O} K$ edges to determine the valence (charge) state of $\mathrm{Fe}$ in $\mathrm{BiFeO}_{3}$. Interestingly, the magnetic measurement results revealed the existence of spin reorientation transition in $\mathrm{Pb}$ and Ti-modified $\mathrm{BiFeO}_{3}$, which indicates that the $\mathrm{BiFeO}_{3}$ samples studied may find promising applications in memory and spintronic devices.
\end{abstract}

\section{Introduction}

A good understanding of the structure-property relationships can be used to develop new functional materials and devices. In recent years, multiferroics have great interest because in these materials, the electrical polarization emerges caused by the symmetry destruction of magnetic structure at magnetic ordering temperature. Rhombohedral distorted perovskite multiferroic $\mathrm{BiFeO}_{3}$ (BFO) prepared under conventional synthesis conditions showed rich variety of subtle interaction among spin, charge, orbital, and lattice degrees of freedom. BFO shows G-type antiferromagnetic spin configuration below to Neel temperature $\left(T_{N}\right)=643 \mathrm{~K}$ and a ferroelectric order at around Curie temperature $\left(T_{C}\right)=$ $1103 \mathrm{~K}[1,2]$. The ferroelectric perovskite $\mathrm{PbTiO}_{3}(\mathrm{PTO})$ is a well-known tetragonal-distorted perovskite, with a space group of $P 4 \mathrm{~mm}, T_{C}=763 \mathrm{~K}$, and a large anisotropic thermal expansion [3]. The tetragonal symmetry is obtained below
$T_{C}$, where PTO belongs to the space group $P 4 m m$, while above $T_{C}$, the cubic $(P m 3 m)$ symmetry describes the system [4]. These perovskite materials have attracted great interest due to their low-cost synthesis, interesting physical properties, and potential applications $[5,6]$. In addition, due to the observation that ultrafast spin rotation times have possible industrial applications, the spin orientation transition (SRT) in antiferromagnetic insulators has attracted the attention of researchers [7-10]. SRTs above and below room temperature have been reported in $\mathrm{BiFeO}_{3}[2,10]$. However, single crystal magnetic and neutron scattering studies have not yet revealed this reorientation transition in $\mathrm{BiFeO}_{3}$ and are therefore questioned $[2,10,11]$.

Bhattacharjee et al. reported a clear indication of SRT in $(1-x)$ BFO- $(x)$ PTO solid solution using magnetization and neutron scattering studies over a small composition range $(0.27<x<0.31) \quad[10]$. The spin reorientation present in $(1-x) \mathrm{BFO}-(x) \mathrm{PTO}$ is different 
from the SRT in $\mathrm{RFeO}_{3}$, but it appears to be Morin transition in hematite. Gaikwad et al. also observed two magnetic anomalies around 124 and $213 \mathrm{~K}$ from low temperature infrared absorption spectra and magnetization data, which are related to the spin reorientation of $\mathrm{Fe}^{3+}$ ions and spin glass state [2]. The local structure of the $\mathrm{FeO}_{6}$ octahedra has distortion and provides a mark for robust spin-phonon coupling in the BFO along with spin reorientation of $\mathrm{Fe}^{3+}$ [2]. Zhu et al. [12] reported the presence of a morphotropic phase boundary (MPB) area in a series of $(1-x) \mathrm{BFO}-x \mathrm{PTO}$ solid solutions. In these solid solutions, the tetragonal phase, rhombohedral phase, and an orthorhombic phase existed at the same time with a large tetragonality in the tetragonal phase segment. The tetragonal symmetry had huge anisotropy $[13,14]$. According to the first principle calculations, the hybridization between the electronic states of cations and anions is crucial to ferroelectricity $[14,15]$. Sati et al. [16] studied the effect of varying $\mathrm{Pr}$ and $\mathrm{Ti}$ codoping concentration on the structural, magnetic, vibrational, and impedance characteristics of $\mathrm{BiFeO}_{3}$ synthesized by the conventional solid-state reaction method. Rietveldrefined XRD patterns indicate that as the doping concentration increases, a compositional driven crystal structure transformation from rhombohedral to an orthorhombic phase existed. Dielectric measurements showed the enhancement in dielectric properties with reduced dielectric loss with increase in doping concentration. Impedance analysis confirmed that with the increase of Pr and Ti concentrations in $\mathrm{BiFeO}_{3}$, the decrease in electrical conductivity was attributed to the enhancement of barrier performance, resulting in the suppression of the lattice conduction path due to lattice distortion. Due to codoping, the magnetic properties of the material are improved, attributed to the breakdown of the balance between antiparallel sublattice magnetization of $\mathrm{Fe}^{3+}$ ions and the collapse of the spatially modulated spin structure caused by the structural transformation [16].

Here, we report the synthesis and analysis of structural and magnetic properties of polycrystalline $\mathrm{Bi}_{1-x} \mathrm{~Pb}_{x} \mathrm{Fe}_{1-x} \mathrm{Ti}_{x} \mathrm{O}_{3}(x=0.05,0.1)$ samples. Rietveld refinement method has been used to analyze the structural parameters. We have been studied the effect of $\mathrm{Pb}$ and $\mathrm{Ti}$ ions doping on structural, vibrational, and magnetic properties in $\mathrm{BiFeO}_{3}$. Polycrystalline ceramics of $\mathrm{Bi}_{1-x} \mathrm{~Pb}_{x} \mathrm{Fe}_{1-x} \mathrm{Ti}_{x} \mathrm{O}_{3}(x=0.05$ and 0.1$)$ are abbreviated as BPFTO-05 and BPFTO-10, respectively, for further communication.

\section{Experimental Details}

The polycrystalline $\mathrm{Bi}_{1-x} \mathrm{~Pb}_{x} \mathrm{Fe}_{1-x} \mathrm{Ti}_{x} \mathrm{O}_{3} \quad(x=0.05$ and 0.1$)$ samples were prepared by the conventional solid-state route. High-purity oxides such as $\mathrm{Bi}_{2} \mathrm{O}_{3}$ (99.99\% purity), $\mathrm{Fe}_{2} \mathrm{O}_{3}$ (99.9\% purity), $\mathrm{PbO}$ (99.99\% purity), and $\mathrm{TiO}_{2}$ (99.99\% purity) were used as starting reagents. Starting reagents were carefully weighed and mixed in stoichiometric ration in an agate mortar for 6 hours using high-purity alcohol as a medium and then calcined at $650^{\circ} \mathrm{C}$ for 6 hours. The leaching process was carried out with distilled water and $\mathrm{HNO}_{3}$ to remove impurities from the samples. The precipitated precursor particles at the bottom were collected, and the excess salts at the top layer were discarded. Pour out the particles repetitively with distilled water and $\mathrm{HNO}_{3}$ to eliminate impurities. Then, the washed particles were dried at room temperature and further calcined at $700^{\circ} \mathrm{C}$ for 5 hours $[17,18]$. XRD measurements were carried out with $\mathrm{CuK} \alpha_{1}(1.5406 \AA)$ radiation using a Bruker D8 Advance $\mathrm{X}$-ray diffractometer and analyzed with the Rietveld refinement method [17]. The Raman spectrum was carried out by "Jobin-Yovn Horiba LABRAM (System HR800) spectrometer with a $488 \mathrm{~nm}$ excitation source equipped with a Peltier cooled CCD detector $(1024 \times 256$ pixels of 26 microns)" [17]. DC magnetization measurements were performed using the physical property measurement system (Quantum Design, PPMS-9). Zero-field cooling (ZFC) and field cooling (FC) processes were used to acquire the temperature dependence of magnetization [19]. XAS was used at normal incidence using the linearly polarized light and in the total electron yield mode at photoelectron station of Beijing Synchrotron Radiation Facility (BSRF), Beijing. The resolution of XAS is $0.3 \mathrm{eV}$ [20].

\section{Results and Discussion}

3.1. Structural Study. Figure 1 shows the $\mathrm{X}$-ray diffraction pattern for $\mathrm{Bi}_{1-x} \mathrm{~Pb}_{x} \mathrm{Fe}_{1-x} \mathrm{Ti}_{x} \mathrm{O}_{3} \quad(x=0.05,0.1)$ samples which are abbreviated as BPFTO-05 and BPFTO-10, respectively. X-ray diffraction data suggest that BPFTO-05 sample possesses rhombohedral structure with the $R 3 c$ space group. All the obtained diffraction peaks completely match the standard crystal data corresponding to JCPDS file 86-1518 [17], while the sample BPFTO-10 shows the mixed phase $(R 3 c, 98 \%+P 4 m m, 2 \%)$ symmetry [21], it matches with the reference data (JCPDS file number 72-1832) completely, except for low-intensity impurity peaks near $2 \theta=27.86^{\circ}$ and $29.04^{\circ}$ related to $\mathrm{Bi}_{2} \mathrm{Fe}_{4} \mathrm{O}_{9}$. The peak splitting is decreasing in BPFTO-10 in comparison to BPFTO-05 [22] results coexistence of two phases. The tetragonal phase had large anisotropy [23]. Thorough important crystallographic parameters acquired through the refinements are given in Table 1.

3.2. Rietveld Refinement of XRD Data. The room temperature XRD patterns of BPFTO-05 and BPFTO-10 ceramics were refined using FullProf software, as shown in Figures 2(a) and 2(b). In the refining process, lattice parameters and profile parameters were refined, while atomic positions and anisotropic displacement parameters were fixed to the values given by earlier reports [24]. The calculated XRD pattern of these two samples are in good agreement with the experimentally observed XRD data with mostly small $R$ values as given in Table 1 . The lattice coordinates of the samples are given in Table 2. For BPFTO-05, the refined lattice parameters such as $a=5.5785 \AA$ and 


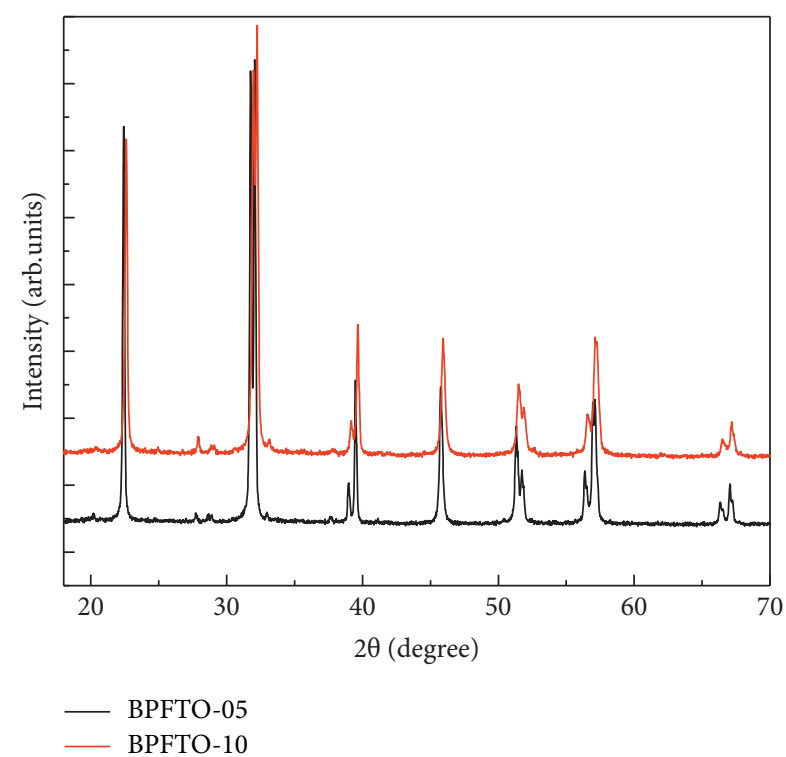

FIgURE 1: X-ray powder diffraction (XRD) pattern of $\mathrm{Bi}_{1-x} \mathrm{~Pb}_{x} \mathrm{Fe}_{1-x} \mathrm{Ti}_{x} \mathrm{O}_{3} \quad(x=0.05$ and 0.1$)$ bulk ceramic at room temperature.

TABLE 1: Details of Rietveld-refined XRD parameters for $\mathrm{Bi}_{1-x} \mathrm{~Pb}_{x} \mathrm{Fe}_{1-x} \mathrm{Ti}_{x} \mathrm{O}_{3}(x=0.05$ and 0.1$)$ samples.

\begin{tabular}{|c|c|c|}
\hline Parameters & $x=0.05$ & $x=0.1$ \\
\hline $2 \theta$ range (deg.) & $20^{\circ}-80^{\circ}$ & $20^{\circ}-80^{\circ}$ \\
\hline Step size (deg.) & $0.02^{\circ}$ & $0.02^{\circ}$ \\
\hline Wavelength & $1.5406 \AA$ & $1.5406 \AA$ \\
\hline $\begin{array}{l}\text { Number of refined } \\
\text { parameters }\end{array}$ & 25 & 21 \\
\hline Space group & $\begin{array}{c}R 3 c \\
(100 \%)\end{array}$ & $\begin{array}{c}R 3 c(99 \%)+P 4 m m \\
(1 \%)\end{array}$ \\
\hline$a(\AA)$ & $5.5865(3)$ & $5.5854(5) / 4.5854(5)$ \\
\hline$b(\AA)$ & $5.5865(3)$ & $5.5854(5) / 4.5854(5)$ \\
\hline$c(\AA)$ & $\begin{array}{c}13.8757 \\
(3)\end{array}$ & $13.8595(3) / 5.9453(4)$ \\
\hline Volume $\left(\AA^{3}\right)$ & $374.88(1)$ & $374.451(1) / 125.021(2)$ \\
\hline$R_{F}$ & 3.68 & $4.05 / 59.1$ \\
\hline$R_{\text {Bragg }}$ & 4.48 & $5.12 / 91.9$ \\
\hline$R_{\mathrm{wp}}$ & 12.4 & 12.0 \\
\hline$R_{\exp }$ & 6.94 & 9.37 \\
\hline$R_{p}$ & 14.3 & 20.0 \\
\hline$x^{2}$ & 2.89 & 1.638 \\
\hline GOF & 1.7 & 1.3 \\
\hline
\end{tabular}

$c=13.8547 \AA$ and crystalline angles, i.e., $\alpha=\beta=90^{\circ}, \gamma=120^{\circ}$, match well with rhombohedral $R 3 c$ symmetry described in another reports $[17,25]$.

3.3. SEM Micrograph Analysis. Figure 3 shows the SEM micrograph for BPFTO-05 and BPFTO-10 samples. SEM micrographs clearly exhibits flakes such as morphology of polycrystalline-prepared sample, and the dopant $\mathrm{Pb}$ and $\mathrm{Ti}$ ions highly influenced the morphology of BFO. Micrograph shows dense structure with nonuniformity in the size of flakes. Also, the fracture type surface in the BPFTO-10 sample was noticeably more transgranular, demonstrating expressively stronger internal stresses, perhaps due to the higher tetragonal distortion for this composition $[22,26]$. For both the samples, an inhomogeneous grain growth was found that resulted in notable residual porosity. The average crystallite size for BPFTO-05 is about $0.8-1 \mu \mathrm{m}$ and for BPFTO- 10 is $0.5-0.8 \mu \mathrm{m}$. The average crystallite size can be reduced by adding $\mathrm{Pb}$ and $\mathrm{Ti}$ ions $\mathrm{BiFeO}_{3}$ attributed to the distinct ionic size of $\mathrm{Pb}^{2+}$ and $\mathrm{Ti}^{4+}$ ions than $\mathrm{Bi}^{3+}$ in $\mathrm{BiFeO}_{3}$.

3.4. XAS Spectra. XAS measurements were performed on both BPFTO-05 and BPFTO-10 samples. Due to large $2 p$ core hole spin-orbit coupling energy, the measured Fe $L_{2,3}$ edge XAS spectra are divided into $L_{3}\left(2 p_{3 / 2}\right)$ and $L_{2}\left(2 p_{1 / 2}\right)$ regions for BPFTO-05 and BPFTO-10, as shown in Figure 4(a) [27]. When Fe ions are located at the $L_{3}$ and $L_{2}$ edges, strong absorption peaks appeared at the photon energies of $709.6 \mathrm{eV}(\mathrm{t} 2 \mathrm{~g})-710.2 \mathrm{eV}(\mathrm{eg})$ and $722 \mathrm{eV}(\mathrm{t} 2 \mathrm{~g})-723.8 \mathrm{eV}(\mathrm{eg})$, respectively. The line shape of $\mathrm{Fe} L_{23}$ edge determines the valence state information of $\mathrm{Fe}$ ions [27]. We observed the prominent presence of XAS signal in $\mathrm{Fe} L_{2,3}$ edge [20]. These results indicate that the samples have the electronic configuration such as $\alpha-\mathrm{Fe}_{2} \mathrm{O}_{3}$ and $\mathrm{LaFeO}_{3}$, and $\mathrm{Fe}^{3+}$ is the leading oxidation states of $\mathrm{Fe}$ ions. The electrostatic interaction is between $\mathrm{O} 2 p$ and $\mathrm{Fe} 3 d$ $t_{2 g}$ and $e_{g}$ orbitals, hybridization of $\mathrm{O} 2 p$ with $B i 6 s / 6 p$ orbitals, hybridization of $\mathrm{O} 2 p$ with $\mathrm{Fe} 4 s / 4 p$ orbitals clearly exists in the prepared systems. Figure 4(b) shows the normalized OK edge XAS spectra for both of these prepared samples, which are evidently showing vacant $\mathrm{O} 2 p$ state in the conduction band [28]. The first two bands denote the hybridization of $\mathrm{O} 2 p$ through unoccupied $\mathrm{Fe} 3 d$ orbitals which splits in $t_{2 g}$ and $e_{g}$ initiated by the electrostatic interaction concerning the $\mathrm{O} 2 p$ and Fe $3 d$ orbitals [28]. Although, another band feature starting at about $\sim 540 \mathrm{eV}$ corresponds to the hybridization of $\mathrm{O} 2 p$ with $\mathrm{Fe} 4 s / 4 p$ orbitals. Improvement in the hybridization of $\mathrm{Bi} 6 s^{2}$ lone pair with $\mathrm{O} 2 p$ orbitals is liable for the enhanced ferroelectric behavior as detected in prepared samples $[20,27,28]$.

3.5. Magnetic Analysis. Temperature dependence zero-field cooling, ZFC (plotted with blue circle), and the field cooling, FC (plotted with red circle), magnetic curves for both BPFTO-05 and BPFTO-10 samples are shown in Figures 5(a) and 5(b). The samples were cooled down to the temperature of $10 \mathrm{~K}$ without an external applied magnetic field in the ZFC mode. Then, we measured the magnetization of the sample with increase in temperature with the applied magnetic field of $0.1 \mathrm{~T}$. Whereas, in the FC mode, the magnetization was measured while cooling down the sample to temperature of $10 \mathrm{~K}$ (as shown in Figures 5(a) and 5(b)). In the FC process, the magnetization reduces with temperature increases. Meanwhile, in the ZFC process, the magnetization takes a magnetic transition around temperature of 160 and $232 \mathrm{~K}$. A noticeable anomaly is observed around $233 \mathrm{~K}$ in the BPFTO-05 sample in the FC mode, which reveals its AFM behavior, and is matched well with previously reported data $[29,30]$. 


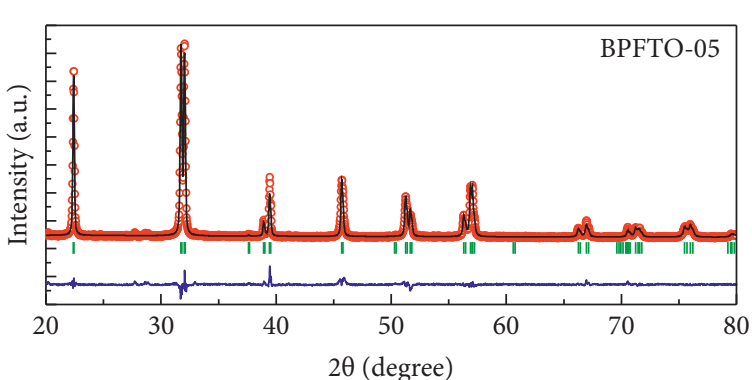

- observed

— calculated

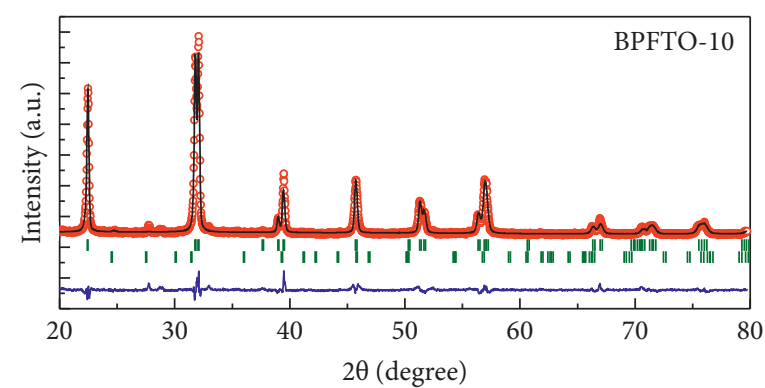

observed - calculated
- difference

I Braggs_positions

(b)

FIGURE 2: Rietveld refined XRD pattern for $\mathrm{Bi}_{1-x} \mathrm{~Pb}_{x} \mathrm{Fe}_{1-x} \mathrm{Ti}_{x} \mathrm{O}_{3}(x=0.05)$ and $\mathrm{Bi}_{1-x} \mathrm{~Pb}_{x} \mathrm{Fe}_{1-x} \mathrm{Ti}_{x} \mathrm{O}_{3}(x=0.1)$ bulk ceramic. The open red circles represent the observed patterns; continuous black and blue color lines represent calculated and difference patterns, respectively. The green tick marks correspond to the position of the allowed Bragg reflections. (a) BPFTO-05. (b) BPFTO-10.

TAвLE 2: Refined structural parameters for $\mathrm{Bi}_{1-x} \mathrm{~Pb}_{x} \mathrm{Fe}_{1-x} \mathrm{Ti}_{x} \mathrm{O}_{3}(x=0.05$ and 0.1$)$ bulk ceramics.

\begin{tabular}{|c|c|c|c|}
\hline & $x$ & $y$ & $z$ \\
\hline \multicolumn{4}{|c|}{$\mathrm{BiFeO}_{3}(R 3 c)[25]$} \\
\hline $\mathrm{Bi}$ & 0.0 & 0.0 & 0.2755 \\
\hline $\mathrm{Fe}$ & 0.0 & 0.0 & 0.0 \\
\hline $\mathrm{O}$ & 0.6679 & 0.7647 & 0.5489 \\
\hline \multicolumn{4}{|c|}{$x=0.05 R 3 c(100 \%)$} \\
\hline $\mathrm{Bi} / \mathrm{Pb}$ & 0.0 & 0.0 & 0.2755 \\
\hline $\mathrm{Fe} / \mathrm{Ti}$ & 0.0 & 0.0 & 0.0 \\
\hline $\mathrm{O}$ & 0.6684 & 0.7648 & 0.5492 \\
\hline \multicolumn{4}{|c|}{$x=0.1 R 3 c(99 \%)+P 4 m m(1 \%)$} \\
\hline $\mathrm{Bi} / \mathrm{Pb}$ & $0.0 / 0.0$ & $0.0 / 0.0$ & $0.2723 / 0.0$ \\
\hline $\mathrm{Fe} / \mathrm{Ti}$ & $0.0000 / 0.5$ & $0.0 / 0.5$ & $0.0 / 0.5973$ \\
\hline O-1 & $-/ 0.5$ & $-/ 0.5$ & $-/ 0.1784$ \\
\hline $\mathrm{O}-2$ & $-1.5575 / 0.5$ & $-0.062 / 0.0$ & $-0.1786 / 0.685$ \\
\hline
\end{tabular}
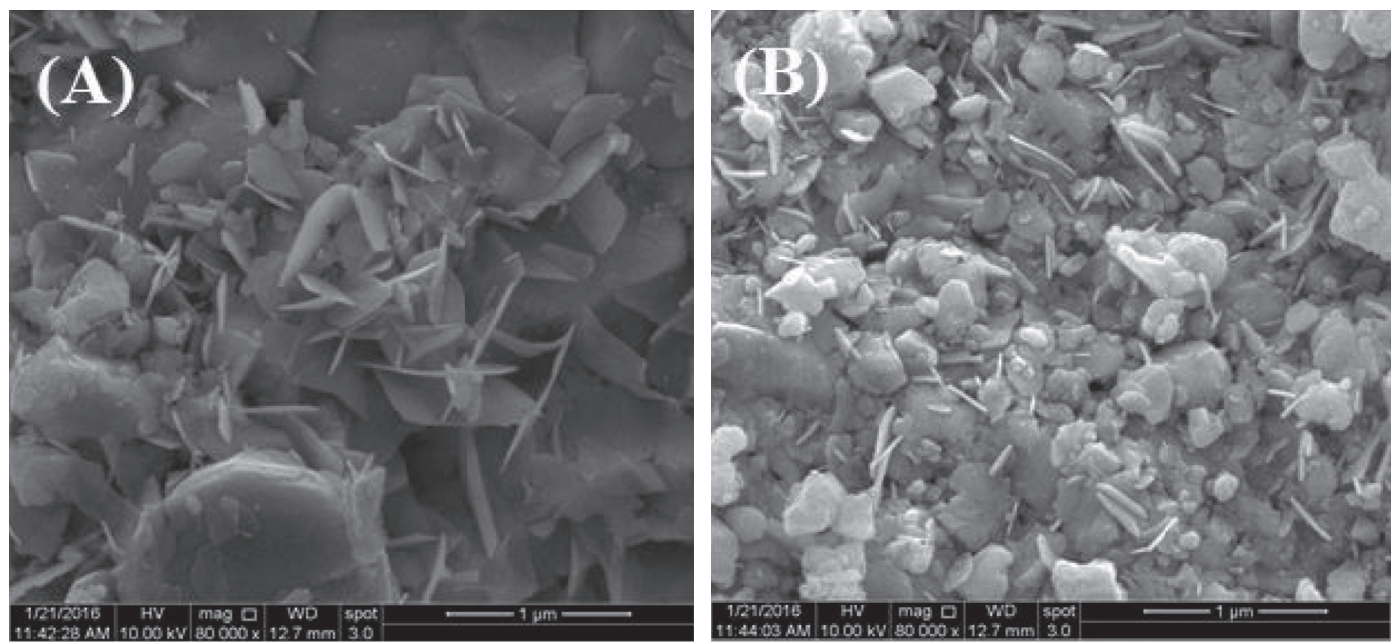

Figure 3: SEM micrograph of $\mathrm{Bi}_{1-x} \mathrm{~Pb}_{x} \mathrm{Fe}_{1-x} \mathrm{Ti}_{x} \mathrm{O}_{3}(x=0.05$ and 0.1$)$ pellet ceramics. (a) $x=0.05$; (b) $x=0.1$.

Another magnetic transition occurs below the temperature of $160 \mathrm{~K}$ in the ZFC mode. The obtained experimental result shows that a change in spin ordering at low temperature attributes to spin reorientation of $\mathrm{Fe}^{3+}$ ions in
$\mathrm{BiFeO}_{3}$ comparable with other rare earth orthoferrites. Though, for bulk BFO single crystal, the spin reorientation has been described close to $50 \mathrm{~K}[2,25,31,32]$. This deviation in $\mathrm{BFO}$ can be attributed to the particle size effect or 


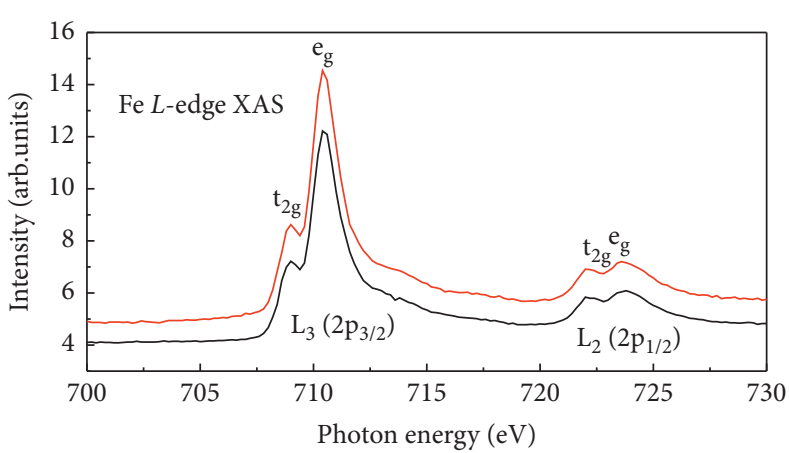

- BPFTO-05

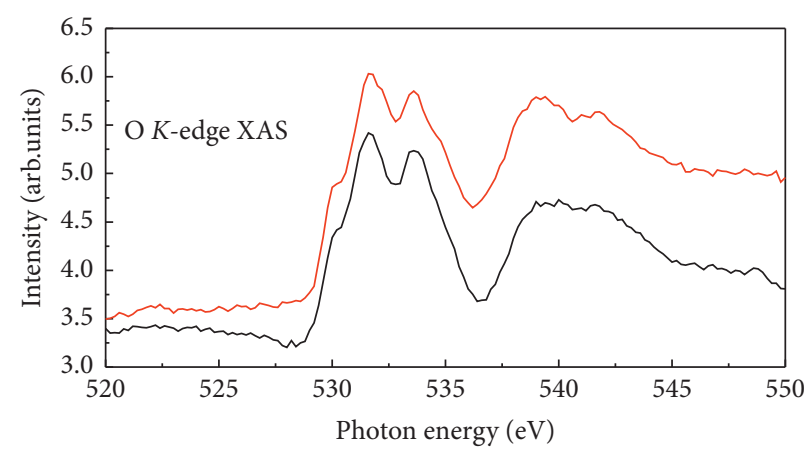

— BPFTO-05

— BPFTO-10

(a)

(b)

FIgURE 4: XAS spectra are divided into (a) $L_{3}\left(2 p_{3 / 2}\right)$ and $L_{2}\left(2 p_{1 / 2}\right)$ regions due to the large $2 p$ core hole spin-orbit coupling energy for BPFTO-05 and BPFTO-10. (b) The OK edge spectrum of BPFTO-05 and BPFTO-10 samples results from excitations to O $2 p$ orbitals interacting with $\mathrm{Fe}$ orbitals.

presence of flakes in SEM micrographs, which is the cause of induced strain, distortion, and lattice disorder. Below $160 \mathrm{~K}$, the magnetization is observed to decrease in the ZFC curve until $50 \mathrm{~K}$ and then increase again until $10 \mathrm{~K}$. However, in the FC curve, no significant change in the magnetization with the decrease in temperature was detected, but it changed around temperature of $232 \mathrm{~K}$. The origin of observed SRT is due to the orientation of $\mathrm{Fe}^{3+}$ spins, and the orientation of $\mathrm{Fe}^{3+}$ spins is caused by the destruction of the antiferromagnetic spiral order. For the BFO system, the magnetic ordering is very complicated due to the Dzyaloshinskii-Moriya (DM) interaction, which leads to a canted AFM ordering of $\mathrm{Fe}^{3+}$ spins in the system [2].

According to Gaikwad et al., "the AFM ordering results in rotation of spins, and the order parameter of this helical ordering is $62 \mathrm{~nm}$. The particles having size less than $62 \mathrm{~nm}$, breaking of the helical ordering of the spins along AFM, are observed. It suppresses the modulated spin structure and improves magnetization" [2]. On increasing the $\mathrm{Pb}$ ion substitution in $\mathrm{BFO}$, there is not a prominent transition like BPFTO-05, but there is little sign of presence of magnetic transitions around temperature of 94,174 , and $224 \mathrm{~K}$ in BPFTO-10. Somehow, we revealed small shift in transition temperature for BPFTO-10 as compared to the BPFTO-05 sample. As it is well known that $\mathrm{BiFeO}_{3}$ is an antiferromagnet and $\mathrm{PbTiO}_{3}$ is diamagnetic, thus as $\mathrm{Pb}$ and $\mathrm{Ti}$ ion increases, it affects the magnetic properties obviously and may vanish the magnetic transitions completely as $\mathrm{PbTiO}_{3}$ is completely ferroelectric material till it is having a tetragonal phase. According to the structural analysis, BPFTO-10 exists simultaneously in two phases rhombohedral and tetragonal near the MPB region. Consequently, it is sensible to recommend that each of these phases undergoes an antiferromagnetic ordering at a different temperature, leading to an anomaly on the temperature dependence of magnetic moment [12]. The $T_{N}$ for both phases decreases with the increase in $x$ across the MPB region [12]. The magnetic curve in the ZFC mode decreases first and then increases at spin reorientation region temperature, a behavior distinctly different from the FC magnetic data that continuously decrease with lowering in temperature. This behavior is evocative of weak ferromagnetism (FM) associated with magnetic glassiness or cluster glass (CG) behavior [33].

Figures 5(c) and 5(d) represent the magnetization versus magnetic field at two different temperatures of 10 and $300 \mathrm{~K}$. Magnetization loops recorded at $300 \mathrm{~K}$ show a remnant value of $\sim 0.004 \mu_{\mathrm{B}}$, as shown in Figures 5(c) and 5(d). This weak ferromagnetic moment or canted antiferromagnetic ordering, seeming small when compared with that of other $\mathrm{BiFeO}_{3}$-based compositions such as $\mathrm{Bi}_{0.7} \mathrm{Ba}_{0.3} \mathrm{FeO}_{3}$ [34], may take place within a tetragonal phase allowing lattice transverse softening. We can observe in Figures 5(c) and 5(d) that magnetization loops recorded at $10 \mathrm{~K}$ show a very less remnant value of $\sim 0.001 \mu_{\mathrm{B}}$, for both the samples. Magnetic moments at $10 \mathrm{~K}$ show true antiferromagnetic ordering in both prepared samples. However, possibly the enhancement in the resultant magnetic moment attributed to the broken cycloid spin structure caused by the variation in crystallographic arrangement with $\mathrm{Pb}$ and $\mathrm{Ti}$ doping in $\mathrm{BiFeO}_{3}$ [35-38].

3.6. Raman Analysis. For the vibrational study, we probed the Raman spectroscopy at room temperature for BPFTO-05 and BPFTO-10 samples with an excitation wavelength of $514 \mathrm{~nm}$, as shown in Figure 6. Distorted rhombohedralstructured BFO yields 18 optical phonon modes and which can be summarized using following irreducible representation: $\Gamma_{\text {opt }}=4 \mathrm{~A}_{1}+5 \mathrm{~A}_{2}+9 \mathrm{E}$ [16]. According to the group theory, 13 modes $\left(\Gamma_{\text {Raman, } R 3 c}=4 \mathrm{~A}_{1}+9 \mathrm{E}\right)$ are Raman active, while $5 A_{2}$ modes be Raman inactive $[17,39,40] . A_{1}$ modes are related with $\mathrm{Fe}$ ions and $E$ modes are allied with $\mathrm{Bi}$ ions. The mode positions dependency on parent BFO and doped BFO is given in Table $3[17,39,41]$. In the present work, as obtained, ten Raman active phonon modes of BPFTO-05 and BPFTO-10 samples including $\mathrm{A}_{1}-1, \mathrm{~A}_{1}-2, \mathrm{~A}_{1}-3$, E-3, E-4, E-5, E-6, E-7, E-8, and E-9 modes at 134 (135), 165 (166), 214 


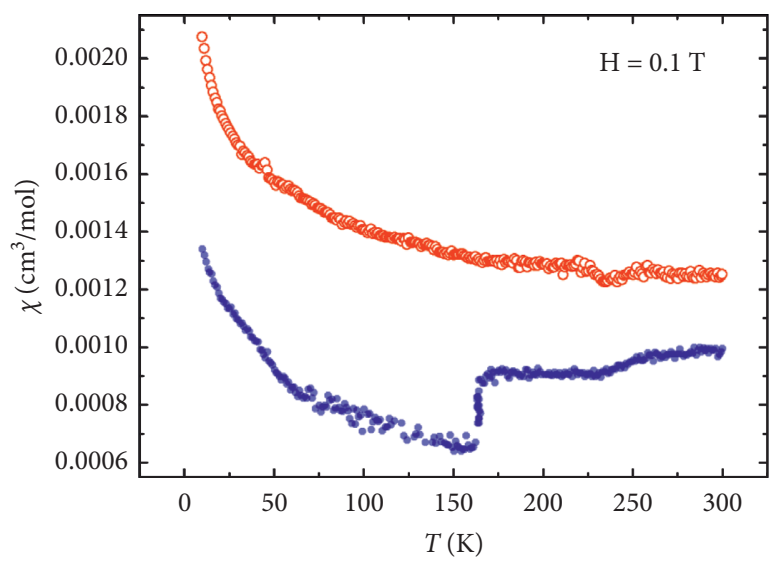

- ZFC

- FC

(a)

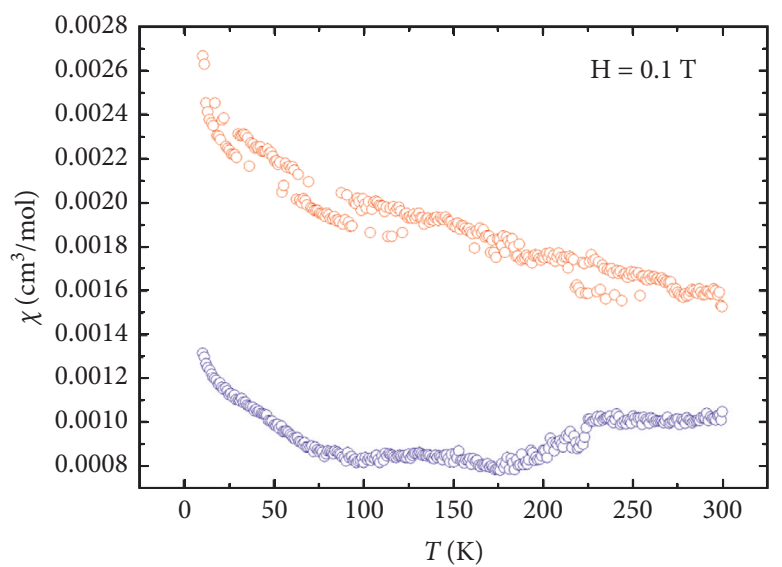

$\circ \mathrm{ZFC}$

$\circ \mathrm{FC}$

(c)

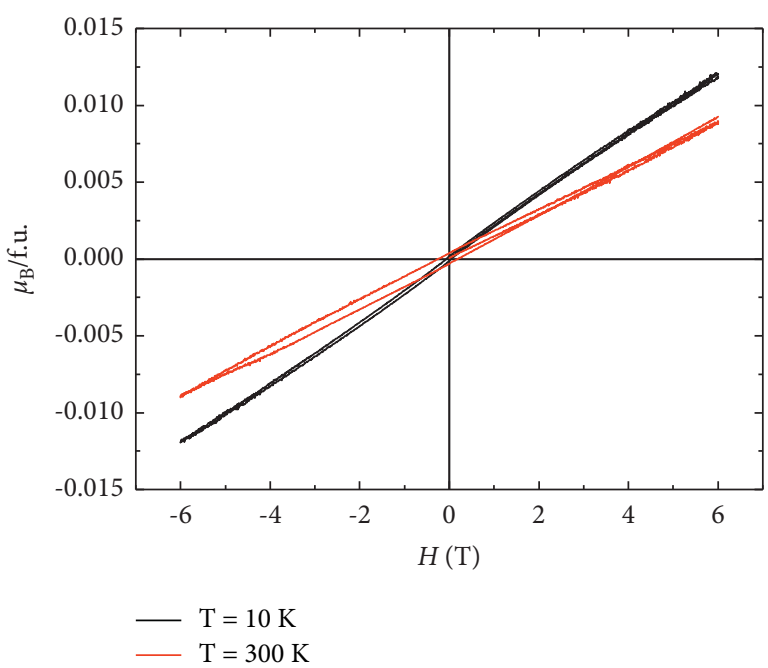

(b)

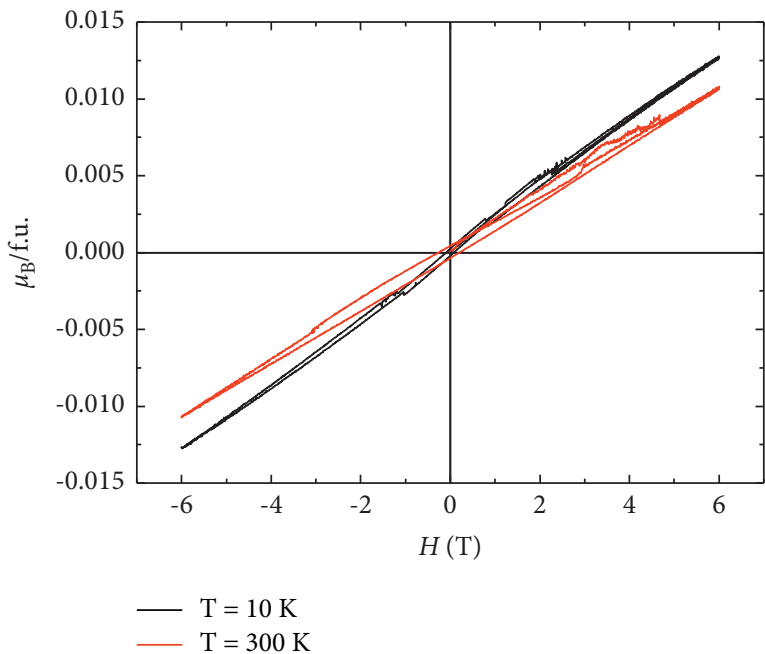

(d)

Figure 5: Temperature dependence of ZFC and FCC magnetization curve for (a) BPFTO-05, (b) BPFTO-10, (c) M-H loop for BPFTO-05, and (d) BPFTO-10.

(216), 269 (264), 308 (309), 368 (362), 475 (470), 514 (524), $542(542)$, and $624(625) \mathrm{cm}^{-1}$ are in good agreement with earlier reported data [41, 42]. The stereochemical activity of the $\mathrm{Bi}$ ion lone pair electron which is principally responsible for the change in both $\mathrm{Bi}-\mathrm{O}$ covalent bonds originates ferroelectricity in $\mathrm{BFO}$. It is believed that the six characteristic modes, i.e., E-1, $A_{1}-1, A_{1}-2, A_{1}-3, A_{1}-4$, and $E-2$ are accountable for the ferroelectric nature of the $\mathrm{BFO}$ samples [17]. As evident from the X-ray diffraction, the crystal symmetry contains rhombohedral $(R 3 c)$ and (rhombohedral $(R 3 c)+$ tetragonal phase $(P 4 \mathrm{~mm}))$ on subsequent doping of $\mathrm{Pb}$ ion and $\mathrm{Ti}$ ion at $\mathrm{A}$-site and $\mathrm{B}$-site of BFO samples, and there are changes in Raman modes as compared to BFO.

These alterations in crystallographic structure is ascribed the A-site and $\mathrm{B}$-site disorder created by $\mathrm{Pb}$ and $\mathrm{Ti}$ ion substitution, "which leads to the shifting of Raman modes at higher and lower frequencies" with sudden disappearance of mode (E-8 in BPFTO-05 and A1-4, E-1, and E-2 in both of the prepared samples) [17]. Additional E-1 and E-2 modes at 68 and $75 \mathrm{~cm}^{-1}$ appear in doped BFO samples, but is too weak to detect in the prepared sample. These phenomena reveal the change of $\mathrm{Bi}-\mathrm{O}$ covalent bonds with increasing doping concentration as compared to BFO and induced ferroelectricity. Moreover, a shift in the Raman characteristic modes towards the higher wavenumber is noted with increasing doping concentration in prepared BPFTO ceramics as compared to pure $\mathrm{BFO}$, which are due to $\mathrm{Bi}-\mathrm{O}$ bond vibrations $[43,44]$. This can be attributed to the lower atomic weight of $\mathrm{Pb}$ (207.2) compared to $\mathrm{Bi}^{+3}$ (208.98) and $\mathrm{Ti}$ (47.86) compared to $\mathrm{Fe}^{+3}$ (55.84) [17]. Furthermore, for BPFTO-05 and BPFTO-10 samples, the intensity of E-modes increase, whereas $A_{1}$ mode intensity decreases significantly. This might be due to some contractions in unit cell volume of the structural phase as rhombohedral $(R 3 c)$ and (rhombohedral $(R 3 c)+$ tetragonal $(P 4 m m)$ ) in BPFTO-10, respectively, and account for the fact that doping affects the 


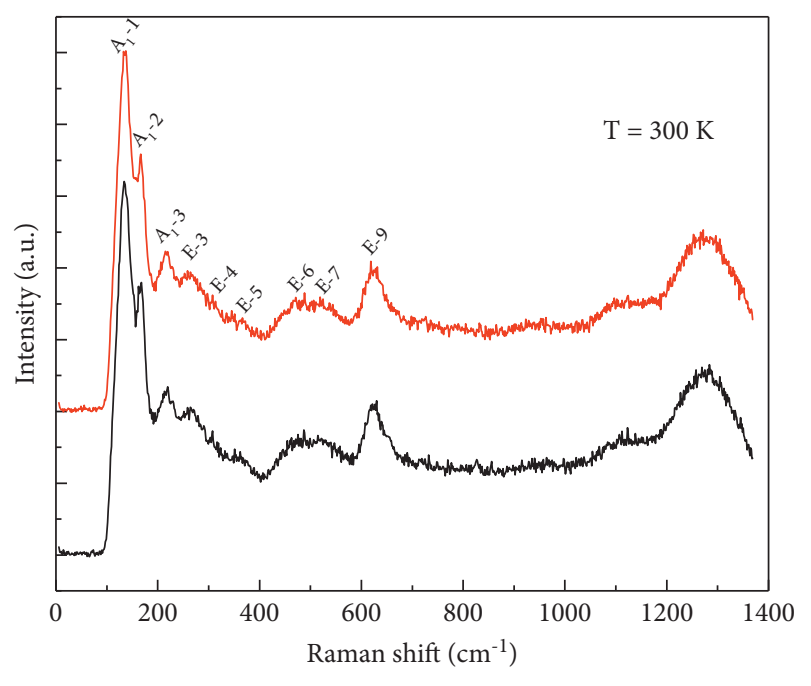

- BPFTO-05

BPFTO-10

FIgURE 6: Raman spectra for $\mathrm{Bi}_{1-x} \mathrm{~Pb}_{x} \mathrm{Fe}_{1-x} \mathrm{Ti}_{x} \mathrm{O}_{3}(x=0.05$ and 0.1 ) at room temperature with excitation wavelength of $514 \mathrm{~nm}$.

TABle 3: Comparison of Raman modes $\left(\mathrm{cm}^{-1}\right)$ from $\mathrm{Bi}_{1-x} \mathrm{~Pb}_{x} \mathrm{Fe}_{1-x} \mathrm{Ti}_{x} \mathrm{O}_{3}(x=0.05$ and 0.1$)$ bulk ceramics and literature.

\begin{tabular}{lccc}
\hline \multirow{2}{*}{ Raman modes $\left(\mathrm{cm}^{-1}\right)$} & \multicolumn{2}{c}{ Present study } & Literature \\
& $x=0.05$ & $x=0.1$ & BFO [39] \\
\hline $\mathrm{A}_{1}-1$ & 165 & 166 & $178(\mathrm{~A})$ \\
$\mathrm{A}_{1}-2$ & 214 & 216 & $229(\mathrm{~A})$ \\
$\mathrm{A}_{1}-3$ & - & - & $502(\mathrm{~A})$ \\
$\mathrm{A}_{1}-4$ & - & - & $591(\mathrm{~A})$ \\
$\mathrm{E}-1$ & - & - & $81(\mathrm{E})$ \\
$\mathrm{E}-2$ & 134 & 135 & $175(\mathrm{E})$ \\
$\mathrm{E}-3$ & 269 & 264 & $242(\mathrm{E})$ \\
$\mathrm{E}-4$ & 308 & 309 & $276(\mathrm{E})$ \\
$\mathrm{E}-5$ & 368 & 362 & $346(\mathrm{E})$ \\
$\mathrm{E}-6$ & 475 & 470 & $368(\mathrm{E})$ \\
$\mathrm{E}-7$ & 514 & 524 & $430(\mathrm{E})$ \\
$\mathrm{E}-8$ & - & 542 & $468(\mathrm{E})$ \\
$\mathrm{E}-9$ & 624 & 625 & $616(\mathrm{E})$ \\
\hline
\end{tabular}

symmetry in BFO [16]. The two-magnon features are almost invisible in the Raman spectrum, but they are advantageous in this study. We detected the broad peak approximately at 964 (955) $\mathrm{cm}^{-1}$ in BPFTO-05 and BPFTO-10, respectively, in the frequency region of $800-1500 \mathrm{~cm}^{-1}$. The peak position of the scattering frequency of two-magnon may be equal to twice energy of zone boundary magnon estimated from the exchange parameters of prepared ceramics [20]. We have observed peaks at $1107(1108) \mathrm{cm}^{-1}$ and $1279 \mathrm{~cm}^{-1}$ for BPFTO-05 and BPFTO-10 samples. The above explained peaks were assigned as the two-phonon Raman scattering process of the prepared samples [45].

\section{Conclusions}

In conclusion, $\mathrm{Bi}_{1-x} \mathrm{~Pb}_{x} \mathrm{Fe}_{1-x} \mathrm{Ti}_{x} \mathrm{O}_{3} \quad(x=0.05,0.1)$ polycrystalline bulk ceramics were synthesized by the conventional solid-state route. X-ray diffraction along with the Rietveld refinement show that BPFTO-05 and BPFTO10 samples have $(R 3 c)$ and $(R 3 c+P 4 m m)$ symmetry, respectively, which are confirmed by Raman scattering data. Through the magnetic data, we have observed the spin reorientation transition above $100 \mathrm{~K}$. It is necessary to use advanced techniques such as neutron scattering to study the properties of observed phenomena in more detail.

\section{Data Availability}

The data used to support the findings of this study are included within the article and are available from the corresponding author upon request.

\section{Disclosure}

The manuscript is presented in abstract form at the International Conference on Minerals Metallurgy and Materials.

\section{Conflicts of Interest}

The authors declare that they have no conflicts of interest.

\section{Acknowledgments}

The authors acknowledge the financial support from the National Natural Science Foundation of China (11774276) and Key Project of Science and Technology of Sichuan Province (2021YFS0355). Kumar and Sharma acknowledge the Luzhou Vocational and Technical College for providing them start-up research grants and well support and also acknowledge the Luzhou talent work leading group office for job incentive and resettlement subsidies for high-level talents. The authors are grateful to Dr. S. Satapathy for their long-term collaboration and numerous fruitful discussion.

\section{References}

[1] D. I. Woodward, I. M. Reaney, R. E. Eitel, and C. A. Randall, "Crystal and domain structure of the $\mathrm{BiFeO}_{3}-\mathrm{PbTiO}_{3}$ solid solution," Journal of Applied Physics, vol. 94, p. 3314, 2009.

[2] V. M. Gaikwad and S. A. Acharya, "Investigation of spin phonon coupling in $\mathrm{BiFeO} 3$ based system by f," Journal of Applied Physics, vol. 114, no. 19, Article ID 193901, 2013.

[3] J. Hong and D. Vanderbilt, "Mapping the energy surface of $\mathrm{PbTiO3in}$ multidimensional electric-displacement space," Physical Review B, vol. 84, no. 11, Article ID 115107, 2011.

[4] K. Abd elmadjid, F. Gheorghiu, M. Zerdali, M. Kadri, and S. Hamzaoui, "Preparation, structural and functional properties of PbTiO3- $\delta$ ceramics," Ceramics International, vol. 45 , no. 7, pp. 9043-9047, 2019.

[5] A. K. Kalyani, R. Garg, and R. Ranjan, "Competing A-site and $\mathrm{B}$-site driven ferroelectric instabilities in the (1-x)PbTiO3-(x) BiAlO3 system," Applied Physics Letters, vol. 94, no. 20, Article ID 202903, 2009.

[6] S. Aoyagi, A. Sawada, J. Harada et al., "Evidence for Pb-O covalency in tetragonal $\mathrm{PbTiO}_{3}$," Physical Review Letters, vol. 87, Article ID 217601, 2001.

[7] R. Nathas, S. J. Pickart, H. A. Alperin, and P. J. Brown, "Polarized-neutron study of hematite, phys rev 136: a1641; schroeer D and nininger jr RC (1967) Morin transition in 
$\alpha-\mathrm{Fe}_{2} \mathrm{O}_{3}$ microcyrstals," Physical Review Letters, vol. 19, p. $632,1964$.

[8] C. Frandsen and S. Morup, "Spin rotation in $\alpha-\mathrm{Fe}_{2} \mathrm{O}_{3}$ nanoparticles by interparticle interactions," Physical Review Letters, vol. 94, Article ID 027202, 2005.

[9] K. Zhang, K. Xu, X. Liu et al., "Resolving the spin reorientation and crystal-field transitions in $\mathrm{TmFeO} 3$ with terahertz transient," Scientific Reports, vol. 6, no. 1, Article ID 23648, 2016.

[10] S. Bhattacharjee, A. Senyshyn, H. Fuess, and D. Pandey, "Morin-type spin-reorientation transition below the Néel transition in the monoclinic compositions of $(1-x) \mathrm{BiFeO}_{3}$ $x \mathrm{PbTiO}_{3}(x=0.25$ and 0.27$)$ : a combined dc magnetization and x-ray and neutron powder diffraction study," Physical Review B: Condensed Matter, vol. 87, Article ID 054417, 2013.

[11] M. Ramazanoglu, W. Ratcliff, Y. J. Choi, S. Lee, S.-W. Cheong, and V. Kiryukhin, "Temperature-dependent properties of the magnetic order in single-crystal $\mathrm{BiFeO}_{3}$," Physical Review $B$, vol. 83, no. 17, Article ID 174434, 2011.

[12] W.-M. Zhu, H.-Y. Guo, and Z.-G. Ye, "Structural and magnetic characterization of multiferroic $\left(\mathrm{BiFeO}_{3}\right) 1-x$ $\left(\mathrm{PbTiO}_{3}\right) x$ solid solutions," Physical Review B: Condensed Matter, vol. 78, Article ID 014401, 2008.

[13] H. Yabuta, H. Tanaka, T. Furuta et al., "Enhancement of tetragonal anisotropy and stabilisation of the tetragonal phase by $\mathrm{Bi} / \mathrm{Mn}$-double-doping in $\mathrm{BaTiO}_{3}$ ferroelectric ceramics," Scientific Reports, vol. 7, no. 1, Article ID 45842, 2017.

[14] K. K. Mishra, R. M. Sarguna, S. Khan, and A. K. Arora, "x-T phase diagram of La-substituted $\mathrm{BiFeO}_{3}-\mathrm{PbTiO}_{3}$," AIP Advances, vol. 1, no. 3, Article ID 032126, 2011.

[15] R. E. Cohen, "Origin of ferroelectricity in perovskite oxides, nature 358:136-138; benedek NA, fennie CJ (2013) why are there so few perovskite ferroelectrics," Journal of Physical Chemistry C, vol. 117, pp. 13339-13349, 1992.

[16] P. C. Sati, M. Arora, S. Chauhan, M. Kumar, and S. Chhoker, "Structural, magnetic, vibrational and impedance properties of $\mathrm{Pr}$ and $\mathrm{Ti}$ codoped $\mathrm{BiFeO} 3$ multiferroic ceramics," $\mathrm{Ce}$ ramics International, vol. 40, no. 6, pp. 7805-7816, 2014.

[17] A. Kumar and D. Varshney, "Crystal structure refinement of Bi1-xNdxFeO3 multiferroic by the Rietveld method," Ceramics International, vol. 38, no. 5, pp. 3935-3942, 2012.

[18] V. R. Palkar, D. C. Kundaliya, S. K. Malik, and S. Bhattacharya, "Magnetoelectricity at room temperature in theBi0.9-xTbxLa0.1FeO3system," Physical Review B, vol. 69, no. 21, Article ID 212102, 2004.

[19] P. Sharma, Y. Xu, H. Fan et al., "Spin reorientation functionality in antiferromagnetic TmFe1-xInxO3 polycrystalline samples," Journal of Alloys and Compounds, vol. 789, pp. 80-89, 2019.

[20] A. Kumar, P. Sharma, W. Yang, J. Shen, D. Varshney, and Q. $\mathrm{Li}$, "Effect of $\mathrm{La}$ and Ni substitution on structure, dielectric and ferroelectric properties of BiFeO 3 ceramics," Ceramics International, vol. 42, no. 13, pp. 14805-14812, 2016.

[21] C. M. Fernández-Posada, A. Castro, J.-M. Kiat et al., "A novel perovskite oxide chemically designed to show multiferroic phase boundary with room-temperature magnetoelectricity," Nature Communications, vol. 7, no. 1, Article ID 12772, 2016.

[22] J. Cheng, S. Yu, J. Chen, Z. Meng, and L. E. Cross, "Dielectric and magnetic enhancements in $\mathrm{BiFeO} 3-\mathrm{PbTiO} 3$ solid solutions with La doping," Applied Physics Letters, vol. 89, no. 12, Article ID 122911, 2006.

[23] L. F. Cotica, V. F. Freitas, I. B. Catellani, I. A. Santos, D. Garcia, and J. A. Eiras, "High-resolution structural studies and covalent bond interactions in $\mathrm{BiFeO} 3-$
PbTiO3compounds: the role of ferroism," Applied Physics Letters, vol. 101, no. 17, Article ID 172903, 2012.

[24] A. Basu, R. Jana, R. Ranjan, and G. D. Mukherjee, "Pressure effects on model ferroelectricBiFeO3-PbTiO3: m," Physical Review B, vol. 93, no. 21, Article ID 214114, 2016.

[25] A. Jaiswal, R. Das, K. Vivekanand, P. Mary Abraham, S. Adyanthaya, and P. Poddar, "Effect of reduced particle size on the magnetic properties of chemically synthesized $\mathrm{BiFeO} 3$ nanocrystals," Journal of Physical Chemistry C, vol. 114, no. 5, pp. 2108-2115, 2010.

[26] J.-R. Cheng, N. Li, and L. E. Cross, "Structural and dielectric properties of Ga-modified $\mathrm{BiFeO}[\mathrm{sub}$ 3]-PbTiO[sub 3] crystalline solutions," Journal of Applied Physics, vol. 94, no. 8, pp. 5153-5157, 2003.

[27] K.-T. Ko, M. H. Jung, Q. He et al., "Concurrent transition of ferroelectric and magnetic ordering near room temperature," Nature Communications, vol. 2, no. 1, p. 567, 2011.

[28] A. Kumar, J. Shen, H. Zhao, Q. Zhengjian, and Q. Li, "Crystallographic and magnetic properties of nanocrystalline perovskite structure SmFeO 3 orthoferrite," Journal of Crystal Growth, vol. 490, pp. 1-5, 2018.

[29] D. M. Giaquinta, G. C. Papaefthymiou, W. M. Davis, and H.-C. Zur Loye, "Synthesis, structure, and magnetic properties of the layered bismuth transition metal oxide solid solution Bi2Fe4-xGaxO9," Journal of Solid State Chemistry, vol. 99, no. 1, pp. 120-133, 1992.

[30] M. G. Rozova, V. V. Grigoriev, I. A. Bobrikov et al., "Synthesis, structure and magnetic ordering of the mullite-type $\mathrm{Bi} 2 \mathrm{Fe} 4-\mathrm{xCrxO} 9$ solid solutions with a frustrated pentagonal Cairo lattice," Dalton Transactions, vol. 45, no. 3, pp. 1192-1200, 2016.

[31] M. K. Singh, W. Prellier, M. P. Singh, R. S. Katiyar, and J. F. Scott, "Spin-glass transition in single-crystalBiFeO3," Physical Review B, vol. 77, no. 14, Article ID 144403, 2008.

[32] A. B. Kuzmenko, D. van der Marel, P. J. M. van Bentum, E. A. Tishchenko, C. Presura, and A. A. Bush, "Infrared spectroscopic study of $\mathrm{CuO}$ : signatures of strong spinphonon interaction and structural distortion," Physical Review B: Condensed Matter, vol. 63, Article ID 094303, 2001.

[33] Z. Qu, Y. Zou, S. Zhang, L. Ling, L. Zhang, and Y. Zhang, "Spin-phonon coupling probed by infrared transmission spectroscopy in the double perovskite Ba2YMoO6," Journal of Applied Physics, vol. 113, no. 17, Article ID 17E137, 2013.

[34] V. A. Khomchenko, D. A. Kiselev, J. M. Vieira et al., "Effect of diamagnetic $\mathrm{Ca}, \mathrm{Sr}, \mathrm{Pb}$, and $\mathrm{Ba}$ substitution on the crystal structure and multiferroic properties of the $\mathrm{BiFeO} 3$ perovskite," Journal of Applied Physics, vol. 103, no. 2, Article ID 024105, 2008.

[35] D. I. Woodward, I. M. Reaney, R. E. Eitel, and C. A. Randall, "Crystal and domain structure of the $\mathrm{BiFeO} 3-\mathrm{PbTiO} 3$ solid solution," Journal of Applied Physics, vol. 94, no. 5, pp. 3313-3318, 2003.

[36] X. Jiang, Y. Jiang, J. Chen et al., "Structural and multiferroic characterization of $\mathrm{BiFeO} 3-\mathrm{PbTiO} 3$-based solid solution with an extra phase," Ceramics International, vol. 44, no. 18, pp. 23315-23319, 2018.

[37] N. Kumar, B. Narayan, A. K. Singh, and S. Kumar, "Enhanced magneto-capacitance in $\mathrm{Sr} 2+$ modified $\mathrm{BiFeO} 3-\mathrm{PbTiO} 3$ solid solutions," Materials Chemistry and Physics, vol. 252, Article ID 123313, 2020.

[38] R. P. Singh and S. Saha, "Additives effect on the multiferroic behaviour of BiFeO3-PbTiO3," Ceramics International, vol. 47, no. 21, pp. 29815-29823, 2021. 
[39] M. K. Singh, H. M. Jang, S. Ryu, and M. H. Jo, "Polarized Raman scattering of multiferroic $\mathrm{BiFeO}_{3}$ epitaxial films with rhombohedral R3c symmetry," Applied Physics Letters, vol. 88, Article ID 42907, 2006

[40] R. Haumont, J. Kreisel, P. Bouvier, and F. Hippert, "Phonon anomalies and the ferroelectric phase transition in multiferroicBiFeO3," Physical Review B, vol. 73, no. 13, Article ID 132101, 2006.

[41] J. Hlinka, J. Pokorny, S. Karimi, and I. M. Reaney, "Angular dispersion of oblique phonon modes in $\mathrm{BiFeO}_{3}$ from microRaman scattering," Physical Review B: Condensed Matter, vol. 83, Article ID 020101, 2011.

[42] R. Palai, H. Schmid, J. F. Scott, and R. S. Katiyar, "Raman spectroscopy of single-domain multiferroic $\mathrm{BiFeO}_{3}$," Physical Review B: Condensed Matter, vol. 81, Article ID 064110, 2010.

[43] L. Luo, W. Wei, X. Yuan, K. Shen, M. Xu, and Q. Xu, "Multiferroic properties of Y-doped BiFeO3," Journal of Alloys and Compounds, vol. 540, pp. 36-38, 2012.

[44] Z. Hu, D. Chen, S. Wang, N. Zhang, L. Qin, and Y. Huang, "Facile synthesis of $\mathrm{Sm}$-doped $\mathrm{BiFeO} 3$ nanoparticles for enhanced visible light photocatalytic performance," Materials Science and Engineering: B, vol. 220, pp. 1-12, 2017.

[45] N. Koshizuka and S. Ushioda, "Inelastic-light-scattering study of magnon softening in ErFeO3," Physical Review B, vol. 22, no. 11, pp. 5394-5399, 1980. 